\title{
EDITORIAL
}

\section{LA PREVENCIÓN SECUNDARIA DE LA ENFERMEDAD CARDIOVASCULAR ES PRIORITARIA PERO RESULTA INSUFICIENTE}

\author{
Fernando Rodríguez Artalejo (1) y Juan del Rey Calero (2)
}

(1) Departamento de Medicina Preventiva y Salud Pública. Universidad del País Vasco. Vitoria-Gasteiz.

(2) Departamento de Medicina Preventiva y Salud Pública. Universidad Autónoma de Madrid. Madrid.

Las limitaciones presupuestarias de los sistemas sanitarios obligan a priorizar las intervenciones sobre la salud, incluidas las preventivas. Dado que a mayor riesgo de enfermar mayor beneficio posible de una intervención, y que el riesgo de un nuevo episodio de enfermedad suele ser mayor en los que ya han padecido uno anterior, las actividades de prevención secundaria suelen considerarse prioritarias. En la práctica, estas actividades compiten, en términos financieros y de tiempo de los profesionales sanitarios para llevarlas a cabo, con las intervenciones de prevención primaria. Este editorial describe algunas dificultades de las actividades de prevención secundaria en nuestro medio, y las ilustra con el control de la colesterolemia para la prevención secundaria de la enfermedad cardiovascular. El principal mensaje de este artículo es que la prevención secundaria debe ser prioritaria, pero resulta insuficiente para controlar una enfermedad si no se acompaña de la prevención primaria. En ausencia de esta última, la prevención secundaria podría, incluso, aumentar la carga demográfica y sanitaria de la enfermedad en la población.

Correspondencia:

Dr. Fernando Rodríguez Artalejo

Departamento de Medicina Preventiva y Salud Pública.

Universidad del País Vasco.

Avda. Universidad, $\mathrm{s} / \mathrm{n}$

01006 Vitoria Gasteiz

Correo electrónico: osproarf@vc.ehu.es
Desde hace tres décadas se sabe que el colesterol plasmático es uno de los principales factores de riesgo cardiovascular. Sin embargo, las evidencias de que la reducción de la colesterolemia disminuye la mortalidad cardiovascular son recientes, en general posteriores a 1994 cuando se publicó el estudio $4 \mathrm{~S}^{1}$. Para la prevención secundaria de las enfermedades cardiovasculares, se sabe que dos estatinas, la simvastatina y la pravastatina, reducen un 20-25\% la colesterolemia y disminuyen un $20-40 \%$ la mortalidad por cardiopatía isquémica $(\mathrm{CI})$, en pacientes que han sufrido un episodio previo de infarto agudo de miocardio, angina estable o angina inestable. Dichos medicamentos reducen el riesgo isquémico en pacientes con niveles de colesterol altos, según se deriva del ensayo $4 S^{1}$, y en pacientes con niveles de colesterol relativamente bajos, según se deriva de los ensayos $\mathrm{CARE}^{2}$ y $\mathrm{LIPID}^{3}$. El tratamiento es seguro, y da lugar a una reducción de la mortalidad general derivada de la disminución de la mortalidad coronaria y cerebrovascular ${ }^{4}$. Además, el tratamiento evita hospitalizaciones e intervenciones de revascularización coronaria. Por último, a mayor riesgo coronario mayor beneficio del tratamiento, pues es menor el número de sujetos que es necesario tratar para evitar un nuevo episodio de enfermedad o muerte.

I a prevención secundaria pretende reducir la aparición de nuevos episodios cardiovasculares, y no solo el control de un factor de riesgo, como 
la colesterolemia. Para conseguirlo se deben controlar los otros factores de riesgo, como el tabaco, la hipertensión arterial y la obesidad. Además, se busca la rehabilitación del paciente para reintcgrarsc a una vida familiar, laboral y social plena; para ello es necesaria la actividad física programada y supervisada. Hay evidencias de que la prevención secundaria multifactorial mejora la supervivencia y la calidad de vida ${ }^{5}$. Finalmente, las intervenciones de prevención secundaria son las prioritarias en la prevención cardiovascular, pues se dirigen a los pacientes que, por tener mayor riesgo, más pueden beneficiarse del tratamiento ${ }^{6}$.

Esta información es relevante para la salud pública, pues las enfermedades cardiovasculares son la primera causa de muerte en nuestro país, y las muertes y hospitalizaciones por las mismas están aumentando en los últimos años y continuarán haciéndolo por el progresivo envejecimiento de la población ${ }^{7}$. Sin embargo, hay dificultades para transformar la información en acción.

En primer lugar, el estudio PREVESE ha puesto de manifiesto que una parte importante de los pacientes que salen de las unidades coronarias de los hospitales españoles no reciben tratamiento hipolipemiante ${ }^{8}$. Es cierto que el estudio PREVESE II se realizó inmediatamente después de la publicación del primero de los estudios en este campo, el estudio 4S, y que los resultados de los ensayos clínicos tardan cierto tiempo en difundirse. Sin embargo, el estudio PREVESE II, no publicado todavía, muestra que, incluso en 1998 más del $60 \%$ de los pacientes coronarios no reciben hipolipemiantes. El entrenamiento de los profesionales sanitarios en la práctica de la llamada «Medicina Basada en la Evidencia» puede contribuir a acelerar la difusión del conocimiento científico y mejorar su aplicación. A ello también pueden servir los programas de gestión de enfermedades, cuya implementación está empezando en nuestro país. Estos programas pretenden acercar la práctica clínica habitual a la considerada ideal (el llamado «patrón oro» del sistema sanitario), mediante la mejora del conocimiento de la enfermedad por los pacientes, el acceso adecuado al sistema, y el cumplimiento correcto de tratamientos consistentes con las mejores evidencias disponibles ${ }^{9}$.

En segundo lugar, aunque la eficacia de los medicamentos hipolipemiantes es importante en términos relativos (reducen la mortalidad general en un 20-30\% a lo largo de 5 años), es sólo modesta en términos absolutos. Según se deriva del estudio $4 \mathrm{~S}$, en el que el beneficio del tratamiento es mayor porque reclutó pacientes con mayor riesgo, es necesario tratar durante 5 años a 30 pacientes para evitar una muerte. Aunque es un tratamiento eficaz, por comparación con otras terapias ampliamente utilizadas en nuestro sistema sanitario, los resultados muestran que 29 de cada 30 pacientes tratados no obtienen un beneficio en términos de reducción de su mortalidad. Y, sin embargo, el coste medio por paciente y año de un tratamiento con simvastatina o pravastatina es 50.000-100.000 pesetas. Están surgiendo nuevas estatinas que pueden ser más coste-efectivas para reducir la colesterolemia ${ }^{10}$, pero todavía no han demostrado su eficacia para reducir la mortalidad cardiovascular o general en ensayos clínicos frente a placebo, de difícil justificación ética en estos momentos ${ }^{11}$. Las nuevas estatinas tampoco han demostrado ser superiores a las primeras en su capacidad de reducir la mortalidad en ensayos clínicos comparativos, de enormes dificultades logísticas por el gran tamaño muestral requerido y con altos costes de realización. La generalización del tratamiento con estatinas a todos los pacientes que pueden beneficiarse, representa un coste difícil de asumir por el sistema sanitario público. Los altos costes de la medicación son una de las principales razones para priorizar su uso en los pacientes con enfermedad coronaria. Aún cuando este tratamiento sea más coste-efectivo que otros ya habituales, su generalización, probablemente, no podrá sufragarse con cambios en los patrones actuales de uso de medicamentos, y requerirá fondos adicionales. 
En tercer lugar, la mejora de la supervivencia de la Cl es claramente deseable para los pacientes y para la sociedad en general, pero se traduce en un aumento del impacto sociosanitario de la enfermedad en la población. Ello se debe a la cronificación de la $\mathrm{CI}$, que prolonga la invalidez y la necesidad de atención sociosanitaria, y al desarrollo de algunas consecuencias de la $\mathrm{CI}$, como el ictus y la insuficiencia cardiaca (IC). Estos fenómenos ya se aprecian en España. En concreto, mientras la mortalidad por las formas agudas de CI está aumentando, la mortalidad por las formas crónicas está disminuyendo. Además las hospitalizaciones por IC han aumentado más del $70 \%$ en los últimos 15 años, hasta representar la primera causa de hospitalización en personas mayores de 65 años en España. De hecho, la IC se está convirtiendo en la epidemia cardiovascular de finales del siglo $\mathrm{xx}^{7}$. Aún cuando hay medicamentos para diferir la aparición de insuficiencia cardiaca en pacientes con cardiopatía isquémica, su impacto beneficioso a nivel poblacional será presumiblemente escaso ${ }^{12}$. En esta tesitura, la única estrategia sensata es acompañar la prevención secundaria de la $\mathrm{Cl}$ de la prevención primaria, es decir, de la intervención sobre sus causas en la población sana, pacientes de mayor riesgo y población general. Incluso, será necesario actuar sobre las causas de los factores de riesgo, la llamada prevención primordial ${ }^{13}$. Si no se hace, las personas acabarán enfermando de forma progresiva, en número creciente por el envejecimiento de la población, poniendo en marcha toda la historia natural de la enfermedad, y engrosando los grupos prioritarios de prevención cardiovascular, cuya atención ya resulta gravosa en la actualidad.

\section{BIBLIOGRAFÍA}

1. Scandinavian Simvastatin Survival Study Group. Randomised trial of cholesterol lowering in 4444 patients with coronary heart disease: the Scandinavian simvastatin survival study (4S). Lancet 1994; 344: 1383-1389.

2. Sacks FM, Pfeffer MA, Moye LA, Rouleau JL, Rutherford JD, Cole TG et al., for the CARE-investigators. The effect of pravastatin on coronary events after myocardial infarction in patients with average cholesterol levels. N Engl J Med 1996; 335: 1001-1009.
3. The Long-Term Intervention with Pravastatin in Ischemic Disease (LIPID) Study Group. Prevention of cardiovascular events and death with pravastatin in patients with coronary heart disease and a broad range of initial cholesterol levels. N Engl J Med 1998; 339: 1349-1357.

4. Crouse JR III, Byington RP, Furberg CD. HMG-CoA reductase inhibitor therapy and stroke risk reduction: and analysis of clinical trials data. Atherosclerosis 1998; 138: 11-24.

5. O'Connor GT, Buring JE, Yusuf S, Goldhaber SZ, Olmstead EM, Paffenbarger RS Jr, et al. An overview of randomised trials of rehabilitation with exercise after myocardial infarction. Circulation 1989; 80: 234-244.

6. Wood DA, De Backer G, Faegerman O, Graham I, Mancia G, Pyörälä K, together with the members of the Task Force. Prevention of coronary heart disease in clinical practice. Recommendations of the second joint task force of European and other societies on coronary prevention. Eur Heart $\mathbf{J}$ 1998; 19: 1434-1503.

7. Rodríguez Artalejo F, Guallar-Castillón P, Banegas Banegas JR, Rey Calero J. Trends in hospitalization and mortality for heart failure in Spain, 1980-1993. Eur Heart J 1997; 18: 1771-1779.

8. Velasco JA, Cosín J, Lopez-Sendon JL, de Teresa E, de Oya M, Carrasco JL, et al. La prevención secundaria del infarto de miocardio en España. Estudio PREVESE. Rev Esp Cardiol 1997; 50: 406-415.

9. Rodríguez Artalejo F, Banegas Banegas JR, Guallar-Castillón P, Hernández Vecino R. Los programas de gestión de enfermedades y su aplicación a la enfermedad cardiovascular. Med Clin (Barc) 1999. En prensa.

10. Huse DM, Russell MW, Miller JD, Kraemer $\mathrm{DL}$, D'Agostino RD, Ellison $\mathrm{RC}$, et al. Cost-effectiveness of statins. Am J Cardiol 1998; 82: 1357-1363.

11. Furberg CD. Natural statins and stroke risk Circulation 1999; 99: 185-188.

12. Gheorghiade M, Bonow RO. Chronic heart failure in the United States. A manifestation of coronary artery disease. Circulation 1998; 97: 282-289.

13. Rose G. The strategy of preventive medicine. Oxford: Oxford University Press, 1992. 